\title{
Accelerated Life Reliability Evaluation of Grating Ruler for CNC Machine Tools Based on Competing Risk Model and Incomplete Data
}

\section{Haiji Yang}

Ministry of Education, Jilin University

\section{Guofa Li}

Ministry of Education, Jilin University

Jialong He ( $\nabla$ hejl@jlu.edu.cn )

Jilin University

\section{Yupeng Ma}

Beihang University

\section{Liding Wang}

Ministry of Education, Jilin University

\section{Wei Zhang}

Ministry of Education, Jilin University

\section{Research Article}

Keywords: Grating ruler, Reliability evaluation, Accelerated life test, Competing risk model, Incomplete data

Posted Date: May 13th, 2021

DOl: https://doi.org/10.21203/rs.3.rs-502429/v1

License: (9) This work is licensed under a Creative Commons Attribution 4.0 International License. Read Full License

Version of Record: A version of this preprint was published at The International Journal of Advanced Manufacturing Technology on July 13th, 2021. See the published version at https://doi.org/10.1007/s00170-021-07627-w. 


\title{
Accelerated life reliability evaluation of grating ruler for CNC machine tools based on competing risk model and incomplete data
}

\author{
Haiji Yang ${ }^{12}$, Guofa $\mathrm{Li}^{12}$, Jialong He ${ }^{12}$, Yupeng Ma ${ }^{3}$, Liding Wang ${ }^{2}$, Wei Zhang ${ }^{2}$
}

\begin{abstract}
Grating ruler is the high-precision linear displacement sensor used in the servo control system of CNC machine tools. It is one of the key components affecting the machining accuracy of CNC machine tools. Due to the long life-time of grating ruler, and it will cost a lot of time and resources to evaluate its reliability level by traditional life test. At the same time, there are some incomplete data in the failure data, which will cause inaccurate evaluation results. In this paper, an accelerated life reliability evaluation method of grating ruler based on competing risk model and incomplete data is proposed. Firstly, according to the characteristics of grating ruler, the life distribution model based on competitive risk is established, and the representation method and conversion method of failure data considering incomplete data are proposed. Secondly, the accelerated life test system which can apply temperature and humidity accelerated stress is built, and the reliability level of JFT series closed absolute grating ruler is evaluated by the proposed method. Finally, the proposed method is compared with the single life distribution model to verify the effectiveness.
\end{abstract}

Keywords Grating ruler, Reliability evaluation, Accelerated life test, Competing risk model, Incomplete data

\section{Introduction}

Grating ruler is the high-precision linear displacement sensor which integrates mechanical, optical, electronic technology, which is widely used in all kinds of CNC machine tools [1-3]. Grating ruler has the ability to measure high precision displacement, and can quickly feedback the precise position of feed shaft to the CNC system. It is an important part of the closed-loop servo control system of CNC machine tools, and also an important factor affecting the machining accuracy of CNC machine tools $[4,5]$. Therefore, it is of great significance to accurately evaluate the reliability level of grating ruler for improving the machining accuracy of $\mathrm{CNC}$ machine tools [6-8]. Engineers in manufacturing enterprises usually carry out life test on grating ruler. The traditional life test is to drive the reading head of grating ruler to run continuously at a certain speed until failure occurs [9].

$\square$ Jialong $\mathrm{He}$

hej1@jlu.edu.cn

yupeng Ma

mayp14@buaa.edu.cn

${ }^{1}$ Key Laboratory of CNC Equipment Reliability, Ministry of Education, Jilin University, Changchun 130025, China

${ }^{2}$ School of Mechanical and Aerospace Engineering, Jilin University, Changchun, 130025 China

${ }^{3}$ School of Reliability and Systems Engineering, Beihang University, Beijing 100191, China
However, the life of the grating ruler can usually reach more than 10,000 hours, and the traditional life test will consume a lot of time and resources. It is an effective method to quickly evaluate the reliability level of grating ruler by carrying out accelerated life test (ALT) to make grating ruler failure quickly and establishing reliability model of accelerated life process $[10,11]$.

Accelerated life test (ALT) was first proposed by Levenbach in 1957. Its essence is to stimulate the potential failure quickly by improving the stress level of the product, and use the acceleration model to convert the failure data under each stress level into the failure data under normal stress [12]. Accelerated life test can be used to evaluate the reliability of products accurately and efficiently, especially for products with many kinds of stress and complex failure mechanism. After obtaining the failure data, how to establish an accurate reliability model according to the characteristics of the product is the key problem [13-16]. To solve this problem, many scholars have carried out a series of research. Lee et al. analyzed a failure mode and failure mechanism based on field failure, and carried out the accelerated life test of the tripod shaft installed on the power bogies of high-speed train. The torsional fatigue life of tripod shaft is verified by the failure data under three stress levels, and the shape parameters and scale parameters of Weibull distribution model are obtained [17]. Dang et al. carried out accelerated life test for both standard and reduced-wall high-stress MV TRXLPE 
cables with different insulation thicknesses, and a comprehensive statistical analysis based upon the maximum likelihood method was applied to reveal the life expectancy of reduced-wall high-stress TRXLPE cables [18]. Kalaiselvan et al. carried out accelerated life test for C0G and X7R nano ceramic capacitor. The non-parametric method is used to convert the accelerated condition data into actual condition. Finally, the life of the nano ceramic capacitor under normal stress is calculated [19]. Rodriguez-Picon et al. presented different models for reliability inference of devices affected by more than one accelerating variable, and established the general log linear relationship model. Finally, an example is presented applied to resistances [20]. Liang et al studied the influence of the change of shape parameters of Weibull distribution on the efficiency of step-down-stress accelerated life test. The results show that when shape parameters is relatively small, small failure sample number can be chosen, which will help to shorten test time [21]. The above researches are focused on the single life distribution model, such as Weibull distribution, exponential distribution and so on. However, the structure of the grating ruler is complex, and there are many failure locations and failure modes. Although the single life distribution model can evaluate the overall reliability level of the grating ruler, it cannot describe the life distribution of each subsystem. The essence of competing risk model is the cumulative failure model of series system, which has higher accuracy than single distribution model in describing the life distribution of complex products [22-25]. Zhang et al. proposed to adopt adaptive progressively hybrid censoring scheme in constant-stress accelerated life test, and established a dependent competing risks model using bivariate Birnbaum-Saunders distribution [26]. Yang et al. proposed a reliability evaluation method of motorized spindle based on competitive risk model, which divided failures into catastrophic type or degradation type. The multivariate distribution is constructed by copula function, and the correlation between failure data is determined by hypothesis test [27]. Zhang et al. presented reliability and maintenance models for systems subject to multiple dependent competing failure processes with a changing, dependent failure threshold [28]. Grating ruler is an optical-mechanical \& electrical integrative products. The performance degradation of some parts in each subsystem will lead to a decline in the accuracy of grating rulers, such as bearing and spring in the mechanical system, grating glass and light-emitting diodes in optical system, and IC circuits in electronic system [29]. Therefore, when the accuracy of the grating ruler exceeds the limit, it is difficult to accurately determine the failure location, so these failure data belong to incomplete data. Incomplete data will lead to inaccurate reliability evaluation results.

In this study, a reliability evaluation method of grating ruler accelerated life process based on competing risk model and incomplete data is proposed. The proposed method not only considers the influence of incomplete data on the evaluation results, but also describes the life distribution of each subsystem, which is helpful to put forward some suggestions for the subsequent improvement design of grating ruler.

The rest of this paper is organized as follows. In second 2 , the grating ruler is divided into three subsystems, and the sensitive stress is determined. In section 3 , the life distribution model of grating ruler based on competitive risk is established, and the representation method and conversion method of failure data considering incomplete data are proposed. In section 4, an accelerated life test system which can apply temperature and humidity stress is set up. A step stress accelerated life test of JFT series closed absolute grating ruler is carried out, and its reliability level is evaluated by using proposed method. In section 5, the proposed method is compared with the Weibull distribution model to verify the effectiveness. Finally, the study is summarized in Section 6.

\section{Characteristic analysis}

Taking the closed grating ruler as an example, its structure is mainly composed of ruler shell, grating, seal, reading head and other parts, as shown in Fig. 1. According to the principle of function independence and structure independence, the grating ruler can be divided into mechanical system (MS), optical system (OS) and electronic system (ES). The specific division details are shown in Table 1.

The mechanical system mainly plays the role of positioning and protection. The grating ruler is fixed on the mounting surface of the $\mathrm{CNC}$ machine tools through the 
screw hole on the ruler shell. The ruler shell and the seal play a protective role together, which can effectively prevent cutting fluid, chips and dust from entering the interior of the grating ruler. Mechanical system is mainly subjected to velocity stress and temperature stress. The main failure modes of mechanical system include bearing jamming, declining spring performance and aging seal. The main reason for the bearing jam and the decrease of spring performance is the excessive shock generated by the grating ruler when running at high speed. The main reason for the aging of the seal is the thermal oxidative aging of the rubber caused by temperature stress, which intensifies the wear of the seal when the reading head is cyclically moved.

Moiré fringe is generated when the main grating and the indicating grating in the optical system move relative to each other. The photosensitive element converts Moiré fringe into electrical signals, and then the displacement of the indicating grating relative to the main ruler grating can be obtained after filtering the signals through the electronic system. The optical system and electronic system are mainly affected by temperature stress and humidity stress. Under the combined action of temperature and humidity, water vapor will be produced. When the seal is aged to a certain extent, water vapor will bring pollutants into the ulnar shell, which will cause the wear of grating glass in the optical system. At the same time, water vapor will also enter the chips with slight manufacturing defects, thus causing the performance degradation of electronic components.

To sum up, temperature and humidity are the main stresses that cause grating ruler failures. In this paper, temperature and humidity are selected as acceleration stress and velocity as constant stress.

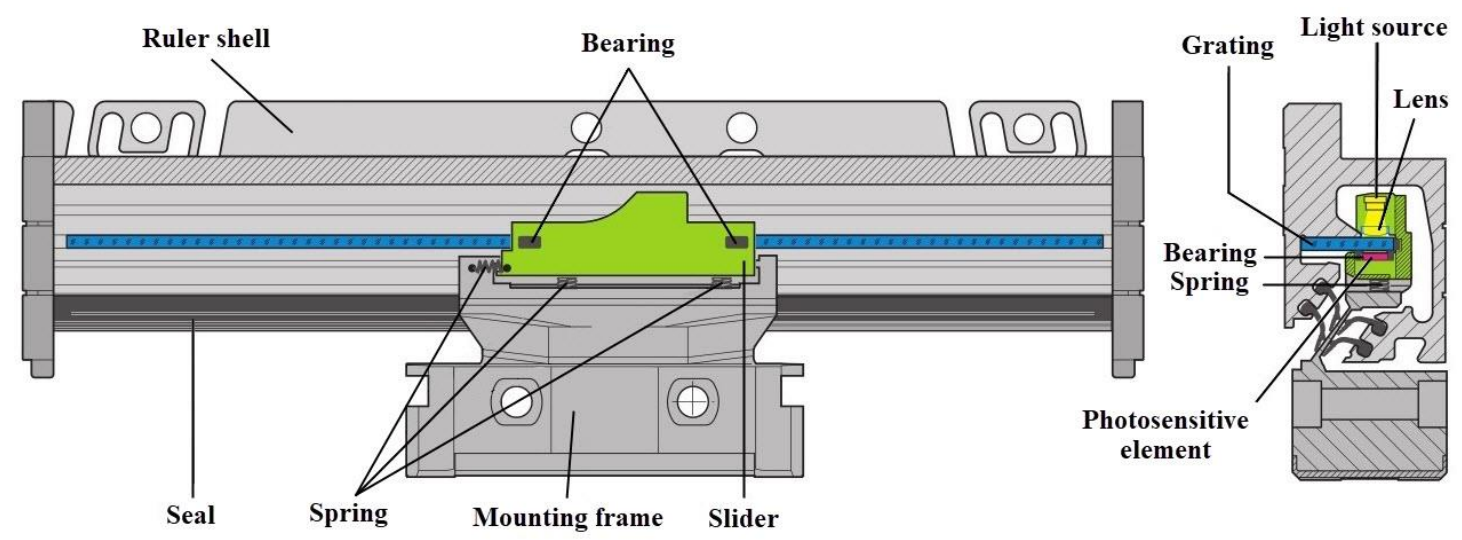

Fig.1 Enclosed grating ruler structure diagram

Table 1 Subsystem division of grating ruler

\begin{tabular}{ll}
\hline Name & Major components \\
\hline Mechanical System & Ruler shell, Seal, Slider, Bearing, Spring etc. \\
& Light source, Main grating, Indicating grating, \\
Optical System & Photosensitive element, etc. \\
& Power circuit, Amplifying circuit, Cable, \\
Electronic System & Digital readout, etc. \\
\hline
\end{tabular}

\section{Life distribution model based on competitive risk}

\subsection{Life distribution model}

Since the competing risk model contains the reliability functions of three subsystems, the subsystem with the highest failure rate of the grating ruler can be observed through the competing risk model, which is convenient for putting forward targeted opinions on the improvement design of the grating ruler. Assuming that $x_{1}, x_{2}$ and $x_{3}$ respectively represent the theoretical life of the mechanical system, optical system and electronic system, then the overall life of the grating ruler can be expressed as [22] :

$T=\min \left(x_{1}, x_{2}, x_{3}\right)$

According to Formula (1), the cumulative failure function of the grating ruler can be determined as:

$$
\begin{aligned}
F(t) & =1-R(t)=1-P(T>t) \\
& =1-P\left(x_{1}>t, x_{2}>t, x_{3}>t\right)
\end{aligned}
$$

Here are five assumptions in the data analysis process of 
the accelerated life test [30].

Hypothesis 1. Unified hypothesis of life distribution model.

Hypothesis 2. The failure mechanism is unchanged.

Hypothesis 3. Characteristic parameter-hypothesis related to accelerated stress.

Hypothesis 4. Cumulative failure assumption.

Hypothesis 5. The failure mechanism is assumed to be statistically independent.

According to Hypothesis 5, the complete failure of the product is only caused by the failure of one subsystem, and the failure time of each subsystem is independent of each other. Formula (2) can be rewritten as:

$F(t)=1-R(t)=1-\prod_{i=1}^{3} P\left(x_{i}>t\right)=1-\prod_{i=1}^{3} R_{i}(t)$

Generally speaking, the life distribution of mechanical system conforms to Weibull distribution. Optical system belongs to optoelectronic system, and its life distribution also conforms to Weibull distribution. The life distribution of electronic system usually conforms to the exponential distribution model. Formula (3) can be rewritten as:

$$
\begin{aligned}
F(t) & =1-R^{(\mathrm{MS})}(t) \cdot R^{(\mathrm{OS})}(t) \cdot R^{(\mathrm{ES})}(t) \\
& =1-\exp \left[-\left(\frac{t}{\eta_{1}^{(\mathrm{MS})}}\right)^{m^{(\mathrm{MS})}}\right] \cdot \exp \left[-\left(\frac{t}{\eta_{1}^{(\mathrm{OS})}}\right)^{m^{(\mathrm{OS})}}\right] \cdot \exp \left(-\frac{t}{\lambda_{1}}\right)
\end{aligned}
$$

where, $m^{(\mathrm{MS})}$ and $\eta_{1}^{(\mathrm{MS})}$ are the shape parameters and characteristic life of Weibull distribution, respectively. $m^{(\mathrm{OS})}$ and $\eta_{1}^{(\mathrm{OS})}$ are the shape parameters and characteristic life of Weibull distribution, respectively. $\lambda_{1}$ is the characteristic life of exponential life distribution.

\subsection{Failure data representation method}

The essence of the competing risk model is the cumulative failure model of the series system, so it is necessary to determine the failure location. However, the performance degradation of some components in each subsystem of the grating ruler will lead to the decline of the accuracy of the grating ruler. Therefore, when the accuracy of the grating ruler exceeds the limit, it is difficult to accurately determine the failure location. These failure data belong to incomplete data. To solve this problem, a traceability variable is added to describe the failure location, and the failure data is specifically expressed as:

$$
x_{i l}=\left[t_{i l}, \varepsilon_{i l}\right], \quad i=1,2, \ldots, n, \quad l=1,2, \ldots, r_{i}
$$

where, $t_{i l}$ is the $l$ th failure time of the $i$ th grating ruler; $\varepsilon_{i l}=$ $\left[\varepsilon_{i l}{ }^{(\mathrm{MS})}, \varepsilon_{i l}{ }^{(\mathrm{OS})}, \varepsilon_{i l}{ }^{(\mathrm{ES})}\right]$ is the traceability variable of failure data. The failure marker value of the system with failure is 1 , and the failure marker value of the system without failure is 0 . The types of traceability variables in failure data can be divided into the following:

I. For complete data, such as failure in a mechanical system, the traceability variable is $\boldsymbol{\varepsilon}_{i l}=[1,0,0]$;

II. For incomplete data, such as the inability to accurately determine whether the failure occurred in mechanical system or optical system, has a traceability variable of $\boldsymbol{\varepsilon}_{i l}=[1,1,0]$.

\subsection{Failure data conversion method}

Since the test stress in the accelerated life test is higher than that under normal working conditions, the failure data under various stress levels need to be converted to the failure data under constant stress [24]. It is assumed that there are $K$ groups of test stresses in the accelerated life test (the first group is normal stress and the other groups are accelerated stress), in which the loading time of the $k$ th stress level $S_{k}$ is $\tau_{k}$, the number of grating ruler is $n$, and there are $r_{i}$ times of failures in the $i$ th grating ruler, in which the occurrence time of the $l$ failure is expressed as $t_{i l}$.

According to Hypothesis 4, the equivalent conversion formula of the cumulative probability density function of Weibull distribution and exponential distribution model is:

$$
\left\{\begin{array}{l}
1-\exp \left\{-\left(\frac{t_{p}^{(p, q)}}{\eta_{q}}\right)^{m}\right\}=1-\exp \left\{-\left(\frac{t_{p}}{\eta_{p}}\right)^{m}\right\} \\
1-\exp \left(-\frac{t_{p}^{(p, q)}}{\eta_{q}}\right)=1-\exp \left(-\frac{t_{p}^{(p, q)}}{\eta_{q}}\right)
\end{array}\right.
$$

where, $\eta_{p}$ and $\eta_{q}$ are the characteristic lives under stress levels $S_{p}$ and $S_{q}$, respectively. Taking Weibull distribution as an example, the effect of equivalent conversion is shown in Fig. 2. 


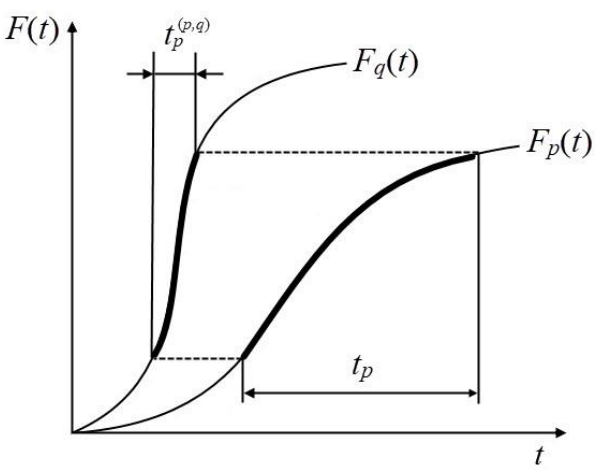

Fig.2 Schematic diagram of equivalent conversion of

Weibull distribution

The accelerated stress in the accelerated life test is temperature and humidity, and the form of the acceleration model is as follows:

$\eta=A \exp \left(\frac{B}{T}\right) \exp \left[\frac{C}{R H}\right]$

where $\eta$ is the characteristic parameter, $A, B, C$ are constants, $T$ is the temperature stress value, and $R H$ is the humidity stress value. After logarithmic processing of the above equation, we can get:

$\ln \eta=a+b \varphi(T)+c \phi(R H)$

where $a=\ln A, b=B, c=C, \varphi(T)=1 / T, \phi(R H)=1 / R H$.

According to Formula (8) and (6), the conversion formula of test time $t_{p}$ under stress $S_{q}$ to equivalent time $t_{p}{ }^{(p, q)}$ under stress $S_{q}$ can be obtained:

$$
\begin{aligned}
t_{p}^{(p, q)} & =\frac{\eta_{q}}{\eta_{p}} t_{p}=t_{p} \frac{A e^{b \varphi\left(T_{q}\right)+c \phi\left(R H_{q}\right)}}{A e^{b \varphi\left(T_{p}\right)+c \phi\left(R H_{p}\right)}} \\
& =t_{p} e^{b\left[\varphi\left(T_{q}\right)-\varphi\left(T_{p}\right)\right]+c\left[\phi\left(R H_{q}\right)-\phi\left(R H_{p}\right)\right]}
\end{aligned}
$$

$$
t_{j}(b, c)=\left\{\begin{array}{l}
t_{i l}^{\left(k_{i l}, 1\right)}=\frac{\eta_{1}^{(*)}}{\eta_{k_{i l}}^{(*)}} \cdot\left(t_{i l}-\sum_{z=1}^{k_{i l}-1} \tau_{z}\right)+\sum_{u=1}^{k_{i l}-1} \frac{\eta_{1}^{(*)}}{\eta_{u}^{(*)}} \tau_{u}, \quad l=1 \\
t_{i l}^{\left(k_{i l}, 1\right)}-t_{i, l-1}^{\left(k_{i, l}, 1\right)}=\frac{\eta_{1}^{(*)}}{\eta_{k_{i l}}^{(*)}} \cdot\left(t_{i l}-\sum_{z=1}^{k_{i l}-1} \tau_{z}\right)-\frac{\eta_{1}^{(*)}}{\eta_{k_{i, l-1}}^{(*)}} \cdot\left(t_{i l}-\sum_{z=1}^{k_{i, l-1}-1} \tau_{z}\right)+\sum_{u=k_{i, l-1}}^{k_{i l}-1} \frac{\eta_{1}^{(*)}}{\eta_{u}^{(*)}} \tau_{u} \\
l=2,3, \mathrm{~L}, r_{i}
\end{array}\right.
$$

where, $*$ is the subsystem symbol, $(*=\mathrm{MS}, \mathrm{OS}, \mathrm{ES})$.

In addition, the censored time should be converted to the censored data of each subsystem under normal stress $S_{1}$. At the end of the accelerated life test (the censored time of stress $\left.S_{K}\right)$, if there are $d$ grating rulers to stop the test, 3(n
Assuming that the $l$ th failure of the $i$ th grating ruler occurs at the $k_{i l}$ stress level, then the conversion time of the occurrence time $t_{i l}$ under the stress $S_{1}$ is:

$t_{i l}^{\left(k_{i l}, 1\right)}=\frac{\eta_{1}}{\eta_{k_{i l}}} \cdot\left(t_{i l}-a_{k_{i l}}\right)+b_{k_{i l}}$

where, $a_{k_{i l}}$ is the sum of the censored time of stress levels from 1 to $k_{i l-1}$, and $b_{k_{i l}}$ is the time of censored time of stress levels from 1 to $k_{i l-I}$ converted to normal stress $S_{1}$, namely:

$$
\begin{aligned}
a_{k_{i l}} & =\tau_{1}+\tau_{2}+\mathrm{L}+\tau_{k_{i l}-1}=\sum_{z=1}^{k_{i l}-1} \tau_{z}, \quad a_{1}=0 \\
b_{k_{i l}} & =\tau_{1}^{(1,1)}+\tau_{2}^{(2,1)}+\mathrm{L}+\tau_{k_{i l}-1}^{\left(k_{i l}-1,1\right)} \\
& =\sum_{u=1}^{k_{i l}-1} \frac{\eta_{1}}{\eta_{u}} \tau_{u}, \quad b_{1}=0
\end{aligned}
$$

According to Formula (11) and (12), the Formula (10) can be transformed into:

$t_{i l}^{\left(k_{i l}, 1\right)}=\frac{\eta_{1}}{\eta_{k_{i l}}} \cdot\left(t_{i l}-\sum_{z=1}^{k_{i l}-1} \tau_{z}\right)+\sum_{u=1}^{k_{i l}-1} \frac{\eta_{1}}{\eta_{u}} \tau_{u}$

According to Formula (9), calculate $\eta_{1} / \eta_{u}$ and $\eta 1 / \eta u$ :

$\left\{\begin{array}{l}\frac{\eta_{1}}{\eta_{k_{i l}}}=e^{b\left[\varphi\left(T_{1}\right)-\varphi\left(T_{k_{i l}}\right)\right]+c\left[\phi\left(R H_{1}\right)-\phi\left(R H_{k_{i l}}\right)\right]} \\ \frac{\eta_{1}}{\eta_{u}}=e^{b\left[\varphi\left(T_{1}\right)-\varphi\left(T_{u}\right)\right]+c\left[\phi\left(R H_{1}\right)-\phi\left(R H_{u}\right)\right]}\end{array}\right.$

According to Formula (13), the time $t_{j}(b, c)(j=1,2, \ldots$, $r$ ) under normal stress $S_{1}$ can be calculated. Assuming that the $j$ th failure data comes from the $l$ th data of the $i$ th grating ruler, then:

$-d$ ) censored data $t_{\tau}$ will be generated. The conversion formula is:

$t_{\tau}^{(K, 1)}=\tau_{1}^{(1,1)}+\tau_{2}^{(2,1)}+\mathrm{L}+\tau_{K}^{(K, 1)}=\sum_{u=1}^{K} \frac{\eta_{1}}{\eta_{u}} \tau_{u}$

$D$ is defined as the serial number set of discarded grating 
ruler, $t_{i(*)}^{(\tau)}(b, c)(i=1,2, \ldots, n, i \notin D)$ is the censored time,

$i$ is the number of the ruler, then:

$t_{i(*)}^{(\tau)}(b, c)=\left\{\begin{array}{l}t_{\tau}^{(K, 1)}=\sum_{u=1}^{K} \frac{\eta_{1}^{(*)}}{\eta_{u}^{(*)}} \tau_{u}, \quad r_{i}=0 \\ t_{\tau}^{(K, 1)}-t_{i, r_{i}}^{\left(k_{\left.i, r_{i}, 1\right)}\right)}=\sum_{u=k_{i, r_{i}}}^{K} \frac{\eta_{1}^{(*)}}{\eta_{u}^{(*)}} \tau_{u}-\frac{\eta_{1}^{(*)}}{\eta_{k_{i, l-1}}^{(*)}} \cdot\left(t_{i, r_{i}}-\sum_{z=1}^{k_{i, r_{i}}-1} \tau_{z}\right), \quad r_{i} \neq 0\end{array}\right.$

where, $*$ is the subsystem symbol, (* = MS, OS, ES).

\subsection{Parameter estimation}

In general, the maximum likelihood estimation (MLE) method is used to estimate the parameters of each life distribution model. However, because the traceability variables in incomplete data belong to hidden variables, it is very difficult to estimate the parameters of models with hidden variables using the maximum likelihood method (MLE) [31]. Expectation maximization algorithm (EM) is an iterative algorithm used to calculate maximum likelihood estimates containing model parameters with hidden variables [32]. The steps of estimating parameters according to EM algorithm are as follows:

Step 1. Build the logarithmic likelihood function:

$$
\begin{aligned}
\ln L\left(\theta \mid x_{j}, t_{i}^{(\tau)}\right)= & \sum_{j=1}^{r}\left[\omega_{j}^{(\mathrm{MS})} \ln f^{(\mathrm{MS})}\left(t_{j}\right)+\omega_{j}^{(\mathrm{OS})} \ln f^{(\mathrm{OS})}\left(t_{j}\right)+\omega_{j}^{(\mathrm{ES})} \ln f^{(\mathrm{ES})}\left(t_{j}\right)\right. \\
& \left.+\left(1-\omega_{j}^{(\mathrm{MS})}\right) \ln R^{(\mathrm{MS})}\left(t_{j}\right)+\left(1-\omega_{j}^{(\mathrm{OS})}\right) \ln R^{(\mathrm{OS})}\left(t_{j}\right)+\left(1-\omega_{j}^{(\mathrm{ES})}\right) \ln R^{(\mathrm{ES})}\left(t_{j}\right)\right] \\
& +\sum_{\substack{i=1 \\
i \notin D}}^{n}\left[\ln R^{(\mathrm{MS})}\left(t_{i}^{(\tau)}\right)+\ln R^{(\mathrm{OS})}\left(t_{i}^{(\tau)}\right)+\ln R^{(\mathrm{ES})}\left(t_{i}^{(\tau)}\right)\right]
\end{aligned}
$$

where, $\theta=\left[m^{(\mathrm{MS})}, m^{(\mathrm{OS})}, a^{(\mathrm{MS})}, b^{(\mathrm{MS})}, c^{(\mathrm{MS})}, a^{(\mathrm{OS})}, b^{(\mathrm{OS})}, c^{(\mathrm{OS})}\right.$, $\left.a^{(\mathrm{ES})}, b^{(\mathrm{ES})}, c^{(\mathrm{ES})}\right] \cdot f^{(*)}(\cdot)$ is the failure probability density function of each subsystem at the initial stress level. $R\left(^{*}\right)(\cdot)$ is the reliability function of each subsystem at the initial stress level. $\omega_{j}^{\left({ }^{*}\right)}$ is the value of the failure marker for each subsystem.

Step 2. Calculate the lower bound function of the expectation step of the $s$ th iteration.

Step 3. Respectively calculate the partial derivatives of lower bound function to each parameter, and obtain an equation set containing 11 equations. Solve the equation set to obtain the estimated values of the parameters.

Step 4. Take lower bound function in step 2 as the initial value of the $s+1$ iteration. Given a minimum positive
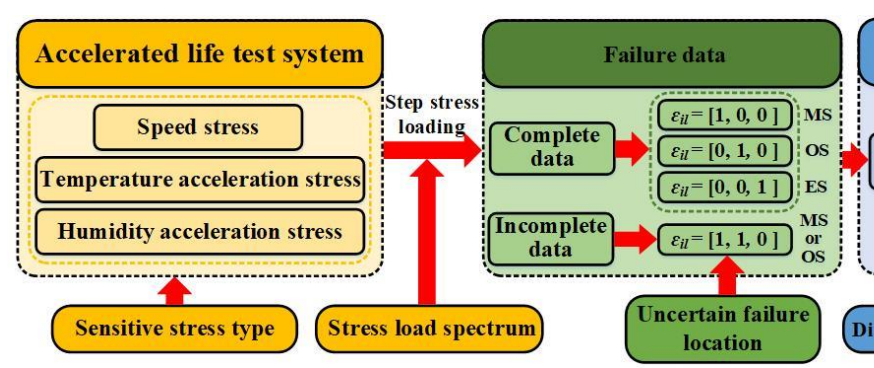

number $\delta$, and determine whether the iteration converges.

\section{Case analysis}

In this section, the proposed method is used to evaluate the reliability level of the closed absolute grating ruler. The tested grating ruler is the JFT series grating ruler produced by Changchun Yu-heng Optical Co., Ltd. (China). Its accuracy is $\pm 5 \mu \mathrm{m}$, resolution is $0.0025 \mu \mathrm{m}$, maximum speed is $180 \mathrm{~m} / \mathrm{min}$, working temperature is 0 to $50^{\circ} \mathrm{C}$, working relative humidity is $20 \%$ to $80 \%$, and sealing grade is IP53. The design distance of the tested grating ruler is $10000 \mathrm{~km}$. In order to improve the test efficiency and reduce the test cost, the step stress loading method was used to carry out the test. The process is shown in Fig. 3.

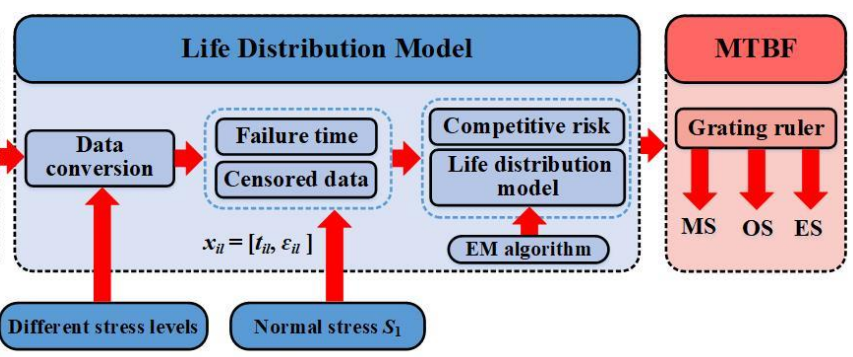

Fig. 3 The process of the case analysis 


\subsection{Accelerated life test system}

The accelerated life test device must be able to load the grating ruler according to the stress type in the actual working condition, and can monitor the relevant parameters in real time [33]. The accelerated life test device includes a servo driving platform and a temperature and humidity test chamber. The servo driving platform is placed inside the temperature and humidity test chamber, as shown in Fig. 4. The servo drive platform is used to load speed constant stress, and the temperature and humidity test chamber is used to load temperature and humidity acceleration stress.

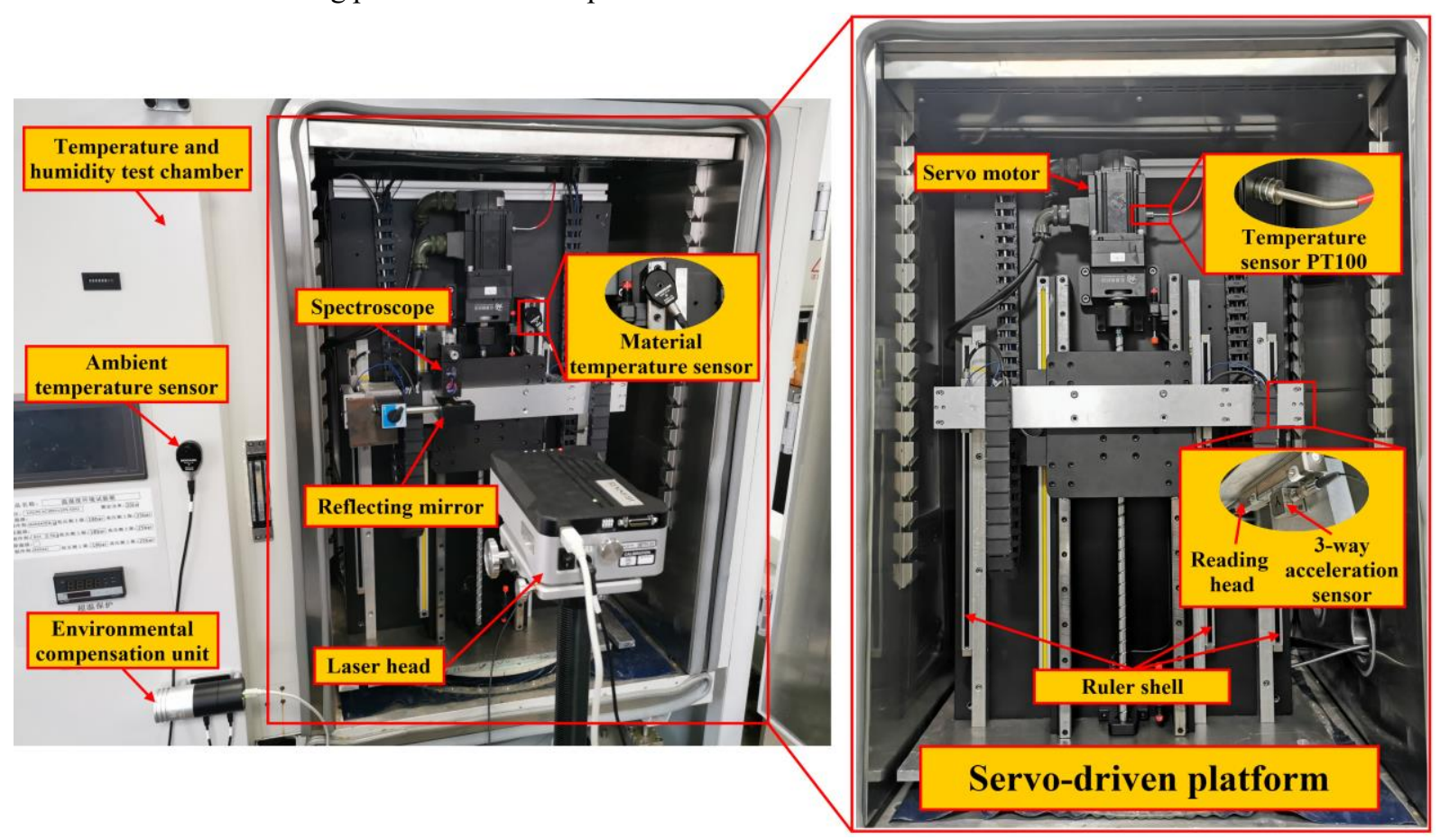

Fig. 4 Accelerated life test device for grating ruler

The servo drive platform has a vertical structure, and the structure of the front and rear sides is the same. The maximum number of test samples is 6. Panasonic A6 series servo motor was selected for the test, with rated speed of $3000 \mathrm{r} / \mathrm{min}$, maximum speed of $5000 \mathrm{r} / \mathrm{min}$ and rated torque of $3.18 \mathrm{~N} \cdot \mathrm{m}$. The model of temperature and humidity test chamber is SUSHI UMC-1200 (China). Its temperature loading range is $-40^{\circ} \mathrm{C}$ to $+150^{\circ} \mathrm{C}$, temperature deviation is $\pm 2^{\circ} \mathrm{C}$, humidity loading range is $20 \%$ to $98 \%$, and temperature deviation is $\pm 5 \%$. The acceleration sensor is placed beside the reading head of the grating ruler. The $\mathrm{Pt}$ 100 temperature sensor is pasted on each servo motor.

The laser interferometer is used to measure the accuracy regularly without removing the grating ruler. The laser interferometer consists of optoelectronic elements such as laser head, spectroscope and reflector; installation elements such as magnetic suction seat, adjusting frame and tripod; compensation elements such as ambient compensation unit and ambient/material temperature sensor.

\subsection{Stress loading scheme}

According to the actual operation data of $\mathrm{CNC}$ machine tool in a gearbox workshop, the 8-level loading spectrum of speed stress is formulated, as shown in Table 2. The distance of the load spectrum is $6 \mathrm{~km}$, the time consuming is $5.4 \mathrm{~h}$, and the average speed is $309 \mathrm{~mm} / \mathrm{s}$.

Table 2 Speed stress loading spectrum

\begin{tabular}{lllll}
\hline Level & $\begin{array}{l}\text { Speed } \\
(\mathbf{m m} / \mathbf{s})\end{array}$ & $\begin{array}{l}\text { Distance } \\
(\mathbf{m m})\end{array}$ & Times & $\begin{array}{l}\text { Proportion } \\
(\boldsymbol{\%})\end{array}$ \\
\hline 1 & 50 & 300 & 6800 & 34 \\
2 & 100 & 300 & 3400 & 17 \\
3 & 150 & 300 & 1400 & 7 \\
4 & 200 & 300 & 2200 & 11 \\
5 & 500 & 300 & 200 & 1 \\
6 & 175 & 300 & 1200 & 6 \\
7 & 125 & 300 & 2000 & 10 \\
\hline
\end{tabular}




\begin{tabular}{lllll}
\hline 8 & 75 & 300 & 2800 & 14 \\
\hline
\end{tabular}

Table 3 Temperature and humidity stress loading scheme

\begin{tabular}{llll}
\hline Level & $\boldsymbol{T}\left({ }^{\circ} \mathbf{C}\right)$ & $\boldsymbol{R H}(\boldsymbol{\%})$ & $\boldsymbol{\tau}(\mathbf{h})$ \\
\hline$S_{1}$ & 25 & 45 & 1125 \\
$S_{2}$ & 33.33 & 56.67 & 1125 \\
$S_{3}$ & 41.67 & 68.34 & 1125 \\
$S_{4}$ & 50 & 80 & 1125 \\
\hline
\end{tabular}

The temperature and humidity stress is the acceleration stress, the upper limit of temperature is $50^{\circ} \mathrm{C}$, the lower limit of $25^{\circ} \mathrm{C}$, the upper limit of humidity is $80 \%$, the lower limit of $45 \%$. The temperature and humidity stress is divided into 4 levels, and $S_{1}$ is the normal stress. The test time of the 4 levels is $\tau_{1}=\tau_{2}=\tau_{3}=\tau_{4}=1125 \mathrm{~h}$, as shown in Table 3 .

\subsection{Evaluation and analysis}

In the process of accelerated life test, if a repairable failure occurs, the test will continue after maintenance, and if an unrepairable failure occurs, the test will be suspended. During the test, a total of 12 failures occurred, of which the accuracy exceeds limit is the unrepairable failure. Failure information is shown in Table 4.

Table 4 Failure information of accelerated life test

\begin{tabular}{|c|c|c|c|c|c|}
\hline NO. & Sample number & Stress level & Failure time & Failure mode & Failure location \\
\hline 1 & 1 & $S_{4}$ & $t_{11}=3681.9$ & Accuracy exceeds limit & Uncertain \\
\hline 2 & 2 & $S_{3}$ & $t_{21}=2797.1$ & Seal damage & Mechanical system \\
\hline 3 & 2 & $S_{4}$ & $t_{22}=4006.5$ & Long grating crack & Optical system \\
\hline 4 & 3 & $S_{2}$ & $t_{31}=1920.3$ & Interface circuit & Electronic system \\
\hline 5 & 3 & $S_{4}$ & $t_{32}=3522.0$ & Bearing stuck & Mechanical system \\
\hline 6 & 3 & $S_{4}$ & $t_{33}=4365.1$ & Accuracy exceeds limit & Uncertain \\
\hline 7 & 4 & $S_{3}$ & $t_{41}=3087.9$ & Scale grating & Optical system \\
\hline 8 & 4 & $S_{4}$ & $t_{42}=4423.8$ & Loose spring & Mechanical system \\
\hline 9 & 5 & $S_{4}$ & $t_{51}=3606.0$ & Power circuit components & Electronic system \\
\hline 10 & 5 & $S_{4}$ & $t_{52}=4289.7$ & Interface circuit & Electronic system \\
\hline 11 & 6 & $S_{4}$ & $t_{61}=3614.5$ & Seal damage & Mechanical system \\
\hline 12 & 6 & $S_{4}$ & $t_{62}=3984.8$ & Scale grating & Optical system \\
\hline
\end{tabular}

According to the method in Section 3.3, the failure data in Table 4 is converted to the data under normal stress $S_{1}$. In the range of possible distribution, define the initial value of the parameter $\hat{\theta}^{(0)}=[5,5,8,25,45,8,25,45,8,25,45]$, and set the iterative convergence condition $\delta=0.000001$. The parameters of the model are calculated as follows:

$\left\{\begin{array}{l}\hat{m}^{(\mathrm{MS})}=4.2417 \\ \hat{a}^{(\mathrm{MS})}=4.0768 \\ \hat{b}^{(\mathrm{MS})}=82.0189 \\ \hat{c}^{(\mathrm{MS})}=100.6566\end{array} \quad\left\{\begin{array}{l}\hat{m}^{(\mathrm{OS})}=3.9790 \\ \hat{a}^{(\mathrm{OS})}=4.5981 \\ \hat{b}^{(\mathrm{OS})}=41.7524 \\ \hat{c}^{(\mathrm{OS})}=152.8763\end{array} \quad\left\{\begin{array}{l}\hat{a}^{(\mathrm{ES})}=5.0501 \\ \hat{b}^{(\mathrm{ES})}=67.7416 \\ \hat{c}^{(\mathrm{ES})}=89.2584\end{array}\right.\right.\right.$

According to Formula (8), the relationship between the characteristic life of each subsystem and the temperature stress and humidity stress can be obtained, as shown in Fig. 5.

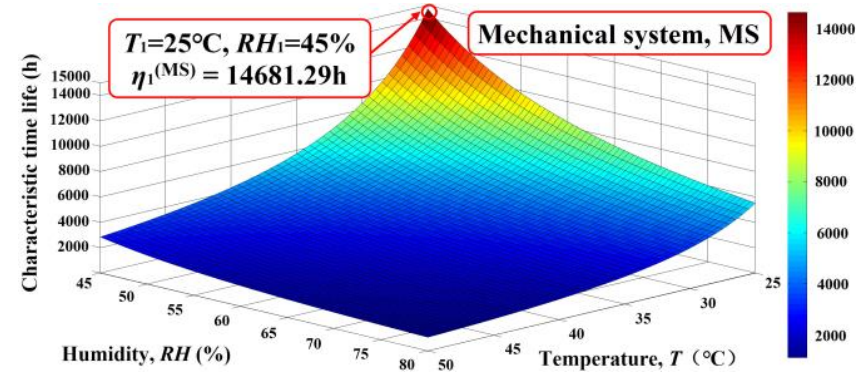

(a) Mechanical system

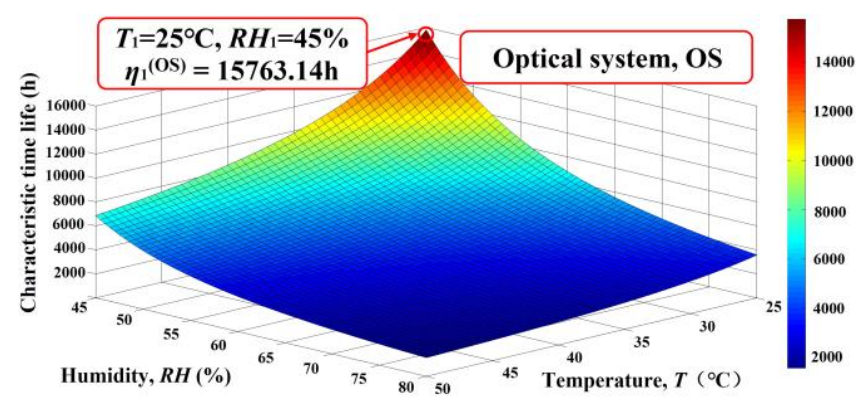

(b) Optical system 


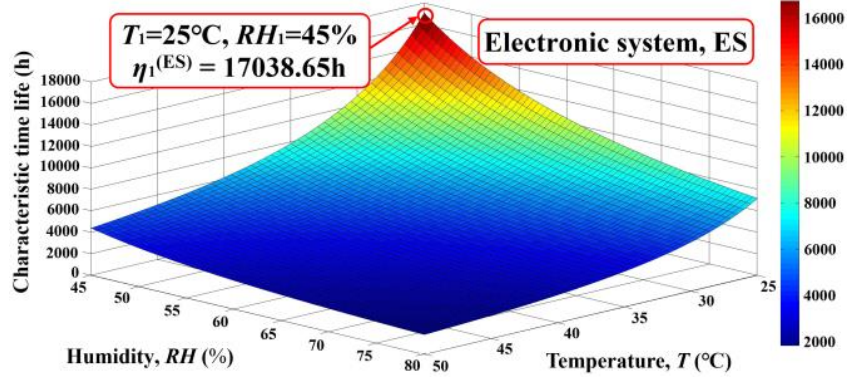

(c) Electronic system

Fig. 5 Relationship between characteristic life and temperature and humidity stress

According to Fig. 5, the characteristic life of each subsystem under normal stress $S_{1}$ can be calculated as $\eta_{1}^{(\text {MS) }}$ $=14681.29 \mathrm{~h}, \eta_{1}^{(\mathrm{OS})}=15763.14 \mathrm{~h}$ and $\eta_{1}^{(\mathrm{ES})}=17038.65 \mathrm{~h}$ respectively.

According to Formula (4), the cumulative probability density function $F(t)$ and the cumulative probability density functions $F^{(\mathrm{MS})}(t), \quad F^{(\mathrm{OS})}(t)$ and $F^{(\mathrm{ES})}(t)$ of the three subsystems can be obtained, as shown in Fig. 6 .

$$
\begin{aligned}
& F(t)=1-\exp \left[-\left(\frac{t}{14681.29}\right)^{4.2417}\right] \cdot \exp \left[-\left(\frac{t}{15763.14}\right)^{3.9790}\right] \\
& \cdot \exp \left(-\frac{t}{17038.65}\right) \\
& \left\{\begin{array}{l}
F^{(\mathrm{MS})}(t)=1-\exp \left[-\left(\frac{t}{14681.29}\right)^{4.2417}\right] \\
F^{(\mathrm{OS})}(t)=1-\exp \left[-\left(\frac{t}{15763.14}\right)^{3.9790}\right] \\
F^{(\mathrm{ESS})}(t)=1-\exp \left(-\frac{t}{17038.65}\right)
\end{array}\right.
\end{aligned}
$$

By deriving $F(t), F^{(\mathrm{MS})}(t), \quad F^{(\mathrm{OS})}(t)$ and $F^{(\mathrm{ES})}(t)$, the probability density distribution function $f(t), f^{(\mathrm{MS})}(t), f^{(\mathrm{OS})}$ $(t)$ and $f^{(\mathrm{ES})}(t)$ are obtained, as shown in Fig. 7.

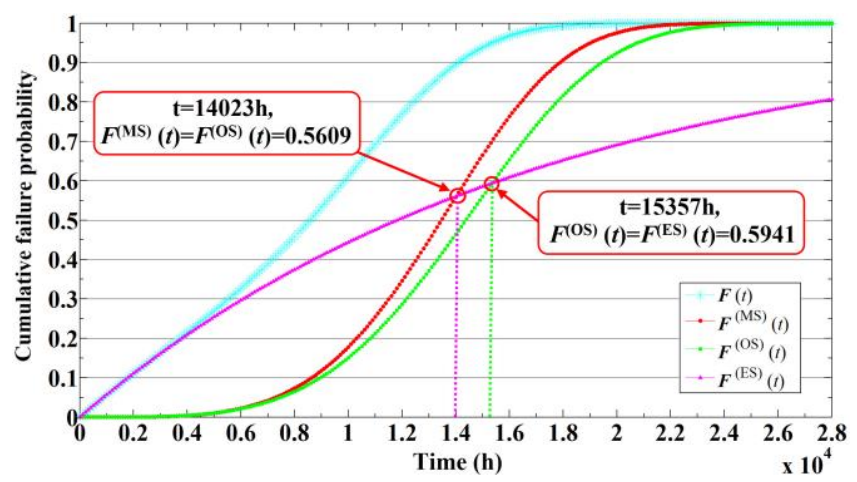

Fig. 6 Cumulative probability density function based on competing risk model

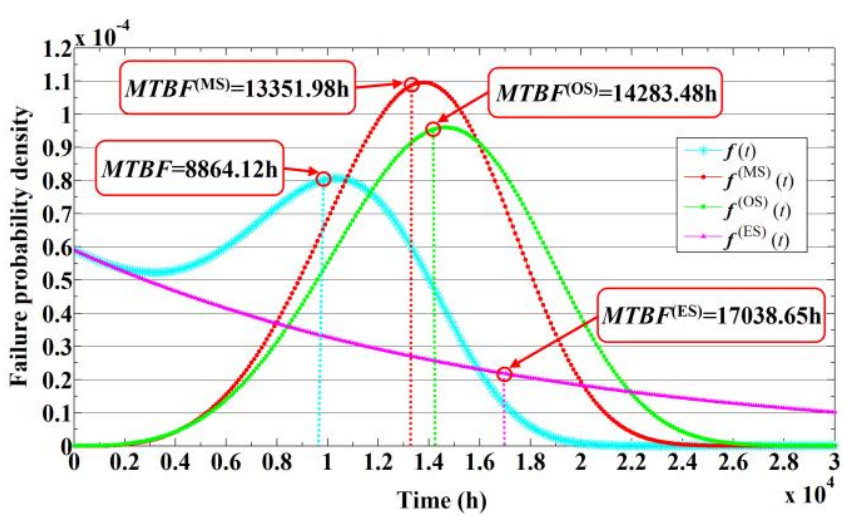

Fig. 7 Probability density function based on competing risk model

$$
\begin{aligned}
& f(t)=\left[0.000289 \cdot\left(\frac{t}{14681.29}\right)^{3.2417}+0.000252 \cdot\left(\frac{t}{15763.14}\right)^{2.979}+0.000059\right] \\
& \cdot \exp \left[-\left(\frac{t}{14681.29}\right)^{4.2417}-\left(\frac{t}{15763.14}\right)^{3.979}-\left(\frac{t}{17038.65}\right)\right] \\
& \left\{\begin{array}{l}
f^{(\mathrm{MS})}(t)=0.000289 \cdot\left(\frac{t}{14681.29}\right)^{3.2417} \cdot \exp \left[-\left(\frac{t}{14681.29}\right)^{4.2417}\right] \\
f^{(\mathrm{OS})}(t)=0.000252 \cdot\left(\frac{t}{17873.01}\right)^{2.979} \cdot \exp \left[-\left(\frac{t}{15763.14}\right)^{3.979}\right] \\
f^{(\mathrm{ES})}(t)=0.000059 \cdot \exp \left[-\left(\frac{t}{17038.65}\right)\right]
\end{array}\right.
\end{aligned}
$$

According to the probability density distribution function $f(t)$, the mean time between failures of grating ruler $\left(M T B F_{t}\right)$ is calculated. At the same time, the mean time between failures of three subsystems $\left(M T B F_{t}^{(\mathrm{MS})}\right.$, $M T B F_{t}^{(\mathrm{os})}, M T B F_{t}^{(\mathrm{ES})}$ ) are calculated, as follows:

$$
\left\{\begin{array}{l}
M T B F_{t}=\int_{0}^{+\infty} t \cdot f(t) d t=8864.12 \mathrm{~h} \\
M T B F_{t}^{(\mathrm{MS})}=\eta_{1}^{(\mathrm{MS})} \cdot \Gamma\left(\frac{1}{\hat{m}^{(\mathrm{MS})}}+1\right)=13351.98 \mathrm{~h} \\
M T B F_{t}^{(\mathrm{OS})}=\eta_{1}^{(\mathrm{OS})} \cdot \Gamma\left(\frac{1}{\hat{m}^{(\mathrm{OS})}}+1\right)=14283.48 \mathrm{~h} \\
M T B F_{t}^{(\mathrm{ES})}=\eta_{1}^{(\mathrm{ES})}=17038.65 \mathrm{~h}
\end{array}\right.
$$

When the average speed is about $309 \mathrm{~mm} / \mathrm{s}, M T B F_{d}=$ $9860.45 \mathrm{~km}, \quad M T B F \underset{d}{(\mathrm{MS})}=14852.74 \mathrm{~km}, \quad M T B F{ }_{d}^{(\mathrm{ES})}$ $=15734.68 \mathrm{~km}$ and $M T B F_{d}^{(0 S)}=18953.79 \mathrm{~km}$.

According to Fig. 6, when $0<t \leq 14023 \mathrm{~h}$, the electronic system with the highest cumulative failure rate and the optical system with the lowest cumulative failure rate. The reason is that most of the early failures of the grating ruler will lead to the abnormality of the digital readout, such as improper installation of the reading head, loosening of the 
fixing screw and poor contact of the plug. At this stage, the electronic system needs key maintenance. When $14023 \mathrm{~h}<\mathrm{t} \leq 15357 \mathrm{~h}$, the cumulative failure rates of the three subsystems are similar. When $t>15357 \mathrm{~h}$, the mechanical system with the highest cumulative failure rate and the electronic system with the lowest cumulative failure rate. The reason is that the precision of some parts in the mechanical system is reduced and the transition wear. At this stage, the mechanical system needs key maintenance. According to Fig 7, compared with optical system and electronic system, the mechanical system has the lowest reliability. In the subsequent design of grating ruler improvement, the mechanical system must be focused on.

\section{Comparison and discussion}

Weibull distribution model is usually used to describe the life distribution of complex electromechanical system. In this section, the proposed method is compared with Weibull distribution model to verify the effectiveness. The parameters of Weibull distribution model are estimated by maximum likelihood estimation [30,31,34]. According to table 4 , the estimated values of parameters calculated as follows:

$\left\{\begin{array}{l}\hat{m}=5.8644 \\ \hat{a}=3.7816 \\ \hat{b}=64.4813 \\ \hat{c}=128.1561\end{array}\right.$

According to Formula (8), the temperature and humidity acceleration model of grating ruler can be obtained:

$\ln \eta=3.7816+\frac{64.4813}{T}+\frac{128.1561}{R H}$

According to the above model, the estimated value of characteristic life under normal stress $S_{1}$ is $\hat{\eta}=9984.26 \mathrm{~h}$. The cumulative probability density function $F(t)$ can be obtained.

$F(t)=1-\exp \left[-\left(\frac{t}{9984.26}\right)^{5.8644}\right]$

The probability density distribution function $f(t)$ can be obtained by deriving $F(t)$.

$f(t)=0.000587 \cdot\left(\frac{t}{9984.26}\right)^{4.8644} \cdot \exp \left[-\left(\frac{t}{9984.26}\right)^{5.8644}\right]$

The mean time between failures $\left(M T B F_{t}\right)$ of the grating ruler can be calculated as:

$M_{T B F_{t}}=\hat{\eta} \cdot \Gamma\left(1+\frac{1}{\hat{m}}\right)=0.9264 \times 9984.26=9249.42 \mathrm{~h}$

When the average speed is $309 \mathrm{~mm} / \mathrm{s}$, the $M T B F_{d}=10289.05 \mathrm{~km}$. The results of the proposed method are $M T B F_{t}=8864.12 \mathrm{~h}, M T B F_{d}=9860.45 \mathrm{~km}$, which is only $4.17 \%$ different from Weibull distribution model. The advantage of the proposed method is that it cannot only describe the overall life distribution of the grating ruler, but also clearly describe the life distribution of each subsystem. It is helpful to find out the subsystem with the highest failure rate in each stage, and put forward targeted suggestions for preventive maintenance and subsequent improvement design.

\section{Conclusion}

Grating ruler is one of the key parts that affect the machining accuracy of CNC machine tools. Because of its long life, it will cost a lot of time and resources to evaluate its reliability level by traditional life test. At the same time, there are some incomplete data in the failure data, which will cause inaccurate evaluation results. To solve the above problems, an accelerated life reliability evaluation method of grating ruler based on competing risk model and incomplete data is proposed. The summary of this study is as follows:

1. The characteristics of the grating ruler are analyzed. According to the different functions, the grating ruler is divided into three subsystems: mechanical system, optical system and electronic system. The sensitive stress of grating ruler is determined, and temperature and humidity are taken as acceleration stress. The life distribution model of grating ruler based on competitive risk is established, and the representation method and conversion method of failure data considering incomplete data are proposed. The model fully considers the influence of incomplete data on the evaluation results, and can describe the life distribution of each subsystem.

2. The accelerated life test system of grating ruler is set up. The $4500 \mathrm{~h}$ step stress accelerated life test of JFT series closed absolute grating ruler is carried out. The results of the proposed method are $M T B F_{t}=8864.12 \mathrm{~h}, M T B F_{d}=$ $9860.45 \mathrm{~km}$, which is only $4.17 \%$ different from Weibull distribution model. Compared with optical system and 
electronic system, the mechanical system has the lowest reliability. In the subsequent design of grating ruler improvement, the mechanical system must be focused on.

\section{Acknowledgements}

This work was supported by the National Natural Science Foundation of China (51905209); Free Exploration Key Project of Natural Science Foundation of Jilin Province Science and Technology Development Plan, China (2020122332JC); Science Research Project of Jilin Province, China(Grant No. 42180); The Fifth Batch of Jilin Young Scientific and Technological Talents Lifting Project(Grant No. QT202114), and Program for JLU Science and Technology Innovative Research Team (JLUSTIRT).

\section{Conflicts of interest}

Not applicable

\section{Availability of data and material}

Not applicable

\section{Code availability}

Not applicable

\section{Authors' contributions}

Haiji Yang: Background research, Data curation, Software, Validation writing-original draft, Editing.

Guofa Li: Methodology, Review \& editing, Supervision.

Jialong He: Supervision, Project administration, Funding acquisition

Yupeng Ma: Review \& editing, Supervision.

Liding Wang: Supervision

Wei Zhang: Assist in experiment, Data curation

All authors read and approved the final manuscript.

\section{Ethics approval}

Not applicable

\section{Consent to participate}

Not applicable

\section{Consent for publication}

Not applicable

\section{Reference}

[1] Fu GQ, Shi JH, Xie YP (2020) Closed-loop mo de geometric error compensation of five-axis ma chine tools based on the correction of axes mov ements. INT J ADV MANUF TECH 110(1-2):3 65-82. https://doi.org/10.1007/s00170-020-05793-x.

[2] Wang Y, Chen HY, Liu ZK (2016) Optical posit ion sensor based on a digital wavelength-encodi ng grating ruler. OPT ENG 55(10): 107103. http s://doi.org/10.1117/1.OE.55.10.107103.

[3] Han WN, Yu P, Li QL (2013) Design of Gratin g Displacement Measuring System Based on Sin gle Chip Microcomputer. APPL MATH MECH 389: 606-611. https://doi.org/10.4028/www.scientif ic.net/AMM.389.606.

[4] Cheng Q, Feng QN, Liu ZF (2016) Sensitivity analysis of machining accuracy of multi-axis ma chine tool based on POE screw theory and Mor ris method. INT J ADV MANUF TECH 84(9):2 301-18. https://doi.org/10.1007/s00170-015-7791-x.

[5] Merghache SM, Hamdi A (2020) Numerical eval uation of geometrical errors of three-axes CNC machine tool due to cutting forces-case: milling. INT J ADV MANUF TECH 111(5-6):1683-705. https://doi.org/10.1007/s00170-020-06211-y.

[6] Cheng Q, Qi BB, Liu ZF (2019) An accuracy d egradation analysis of ball screw mechanism con sidering time-varying motion and loading workin g conditions. MECH MACH THEORY 134:1-23. https://doi.org/10.1016/j.mechmachtheory.2018.12. 024.

[7] Cheng Q, Zhao HW, Zhao YS (2018) Machinin $\mathrm{g}$ accuracy reliability analysis of multi-axis mac hine tool based on Monte Carlo simulation. J I NTELL MANUF 29(1):191-209. https://doi.org/10. 1007/s10845-015-1101-1.

[8] Niu P, Cheng Q, Liu ZF (2021) A machining a ccuracy improvement approach for a horizontal machining center based on analysis of geometric error characteristics. INT J ADV MANUF TEC H 112(9-10):2873-87. https://doi.org/10.1007/s001 70-020-06565-3.

[9] Han D, Bai T (2021) Parameter Estimation Usin g EM Algorithm For Lifetimes From Step-Stress 
and Constant-Stress Accelerated Life Tests With Interval Monitoring. IEEE T RELIAB 70(1):4964. https://doi.org/10.1109/TR.2019.2957710.

[10] Wang LQ, Li YF (2017) Boundary for aviation bearing accelerated life test based on quasi-dyna mic analysis. TRIBOL INT 116:414-421. https:// doi.org/10.1016/j.triboint.2017.06.014.

[11] You DZ, Pham H (2016) Self-adaptive Stress A ccelerated Life Testing Scheme. J BRAZ SOC MECH SCI 39(6):2095-2103. https://doi.org/10.10 07/s40430-016-0683-7.

[12] Zhou W, Wang M, Wu Q (2021) Accelerated Li fe Testing Method of Metallized Film Capacitors for Inverter Applications. IEEE T TRANSP EL ECTR 7(1):37-49. https://doi.org/10.1109/TTE.202 0.3021614 .

[13] Charki A, Laronde R, Guérin F (2011) Robustne ss evaluation using highly accelerated life testin g. INT J ADV MANUF TECH 56(9):1253-61. h ttps://doi.org/10.1007/s00170-011-3264-z.

[14] Ozturk UE (2016) A Novel Approach for Accel erated Life Testing of Ground Vehicle Compone nts. EXP TECHNIQUES 41(2):171-178. https://d oi.org/10.1007/s40799-016-0166-X.

[15] Park M, Rhee S (2018) A Study on Life Evalua tion \& Prediction of Railway Vehicle Contactor Based on Accelerated Life Test Data. J MECH SCI TECHNOL 32(10): 4621-4628. https://doi.or g/10.1007/s12206-018-0909-y.

[16] Wang L, Shi Y (2017) Estimation for constant-st ress accelerated life test from generalized half-n ormal distribution. J SYST ENG ELECTRON 2 8(4):810-6. https://doi.org/10.21629/JSEE.2017.04. 21.

[17] Lee JH, Cho HY (2018) An Experimental Study of the Lifetime of a Tripod Shaft with Torsion al Fatigue Using an Accelerated Life Test Meth od. INT J PRECIS ENG MAN 19(9):1399-1404. https://doi.org/10.1007/s12541-018-0165-1.

[18] Dang C, Cote J, Leblanc P (2018). Accelerated aging test and life expectancy of reduced-wall $\mathrm{h}$ igh-stress MV TRXLPE cables. IEEE T DIELE CT EL IN 25(3):1039-1046. https://doi.org/10.110 9/TDEI.2018.006856.
[19] Kalaiselvan C, Rao LB (2016) Accelerated life t esting of nano ceramic capacitors and capacitor test boards using non-parametric method. Measur ement 88:58-65. https://doi.org/ 10.1016/j.measure ment.2016.03.035.

[20] Rodriguez-Picon LA, Flores-Ochoa VH (2017) E stimation of a log-linear model for the reliability assessment of products under two stress variabl es. Int J Syst Assur Eng Manag 8(2): 1026-104 0. https://doi.org/10.1007/s13198-016-0564-6.

[21] Liang QW, Wand DD (2018) Influence study of Weibull distribution parameter on double stress $d$ own-step accelerated life test. INDIAN J MAR SC I 47(1): 46-52.

[22] Zhao XJ, Xu JY, Liu B (2018) Accelerated Deg radation Tests Planning With Competing Failure Modes. IEEE T RELIAB 67(1):142-155. https://d oi.org/10.1109/TR.2017.2761025.

[23] Yu Z Y, Ren Z Q, Tao J Y (2014) Accelerated Testing with Multiple Failure Modes under Seve ral Temperature Conditions. MATH PROBL EN G, 2014:1-8. https://doi.org/10.1155/2014/839042.

[24] Zhang CF, Shi YM, Wu M (2016) Statistical inf erence for competing risks model in step-stress partially accelerated life tests with progressively Type-I hybrid censored Weibull life data. J CO MPUT APPL MATH 297:65-74. https://doi.org/1 0.1016/j.cam.2015.11.002.

[25] Luo W, Zhang C, Chen X, Tan Y (2015) Accel erated reliability demonstration under competing failure modes. RELIAB ENG SYST SAFE. 201 5;136:75-84. https://doi.org/10.1016/j.ress.2014.11. 014.

[26] Zhang CF, Shi YM, Bai XC (2017) Inference fo r Constant-Stress Accelerated Life Tests With D ependent Competing Risks From Bivariate Birnb aum-Saunders Distribution Based on Adaptive $\mathrm{P}$ rogressively Hybrid Censoring. IEEE T RELIAB 66(1):111-122. https://doi.org/10.1109/TR.2016.26 39583.

[27] Yang ZJ, Li XX, Chen CH (2018) Reliability as sessment of the spindle systems with a competin g risk model. P I MECH ENG O-J RIS 233(2): 226-34. https://doi.org/10.1177/1748006X1877034 
3.

[28] Jiang L, Feng Q, Coit DW (2012) Reliability an d Maintenance Modeling for Dependent Competi ng Failure Processes With Shifting Failure Thres holds. IEEE T RELIAB 61(4):932-48. https://doi. org/10.1109/TR.2012.2221016.

[29] Gurauskis D, Kilikevicius A (2021) Thermal and Geometric Error Compensation Approach for an Optical Linear Encoder. SENSORS-BASEL 21 (2):360. https://doi.org/10.3390/s21020360.

[30] Lin CT, Hsu YY, Lee SY (2019) Inference on c onstant stress accelerated life tests for log-locati on-scale lifetime distributions with type-I hybrid censoring. J STAT COMPUT SIM 89(4): 720-74 9. https://doi.org/10.1080/00949655.2019.1571591.

[31] Fan TH, Wang WL (2011) Accelerated Life Test $\mathrm{s}$ for Weibull Series Systems With Masked Data. IEEE T RELIAB 60(3): 557-569. https://doi.org
/10.1109/TR.2011.2134270.

[32] Ranjan R, Upadhyay SK (2016) Classical and B ayesian Estimation for the Parameters of a Com peting Risk Model Based on Minimum of Expo nential and Gamma Failures. IEEE T RELIAB 6 5(3):1522-1535. https://doi.org/10.1109/TR.2016.25 75439 .

[33] Chen WZ, Yang ZJ, Chen CH (2019) Load-Dep endent Rotating Performance of Motorized Spind les: Measurement and Evaluation Using Multi-Zo nes Error Map. IEEE ACCESS 7: 180482-18049 0. https://doi.org/10.1109/ACCESS.2019.2957599.

[34] Nassar M, Dey S (2018) Different estimation m ethods for exponentiated Rayleigh distribution un der constant-stress accelerated life test. QUAL R ELIAB ENG INT 34(8): 1633-1645. https://doi.o rg/10.1002/qre.2349. 
Figures

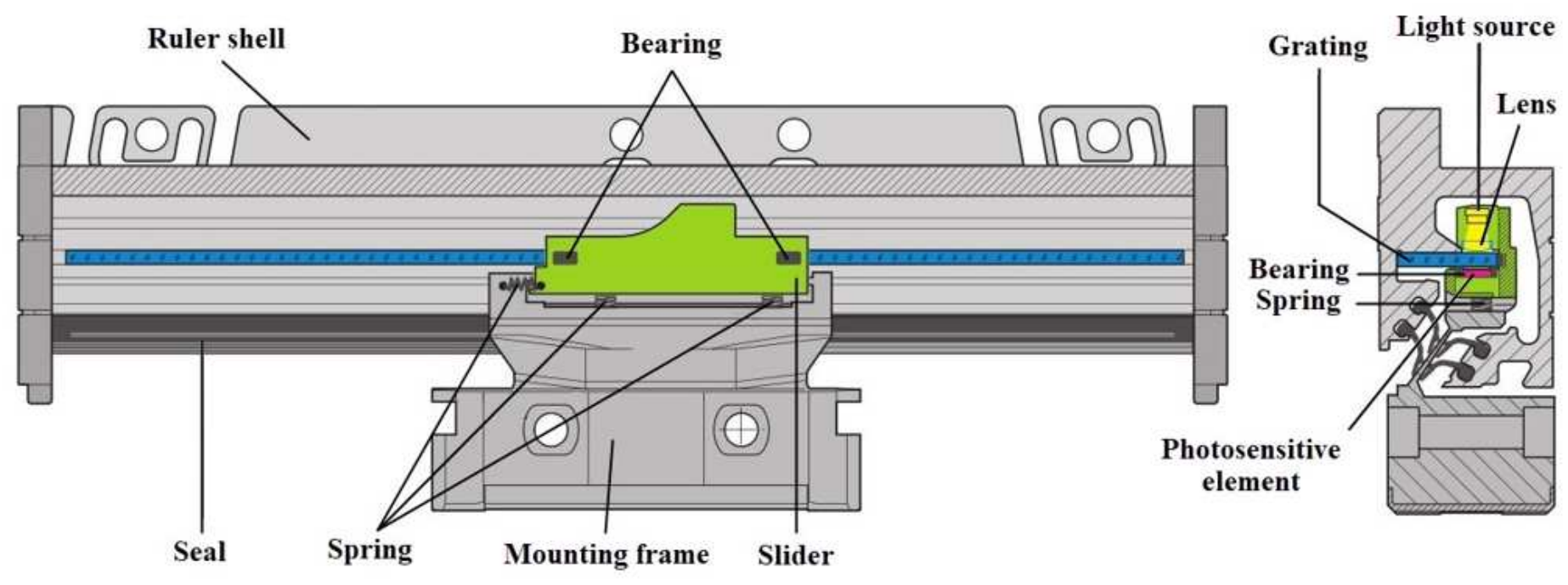

Figure 1

Enclosed grating ruler structure diagram

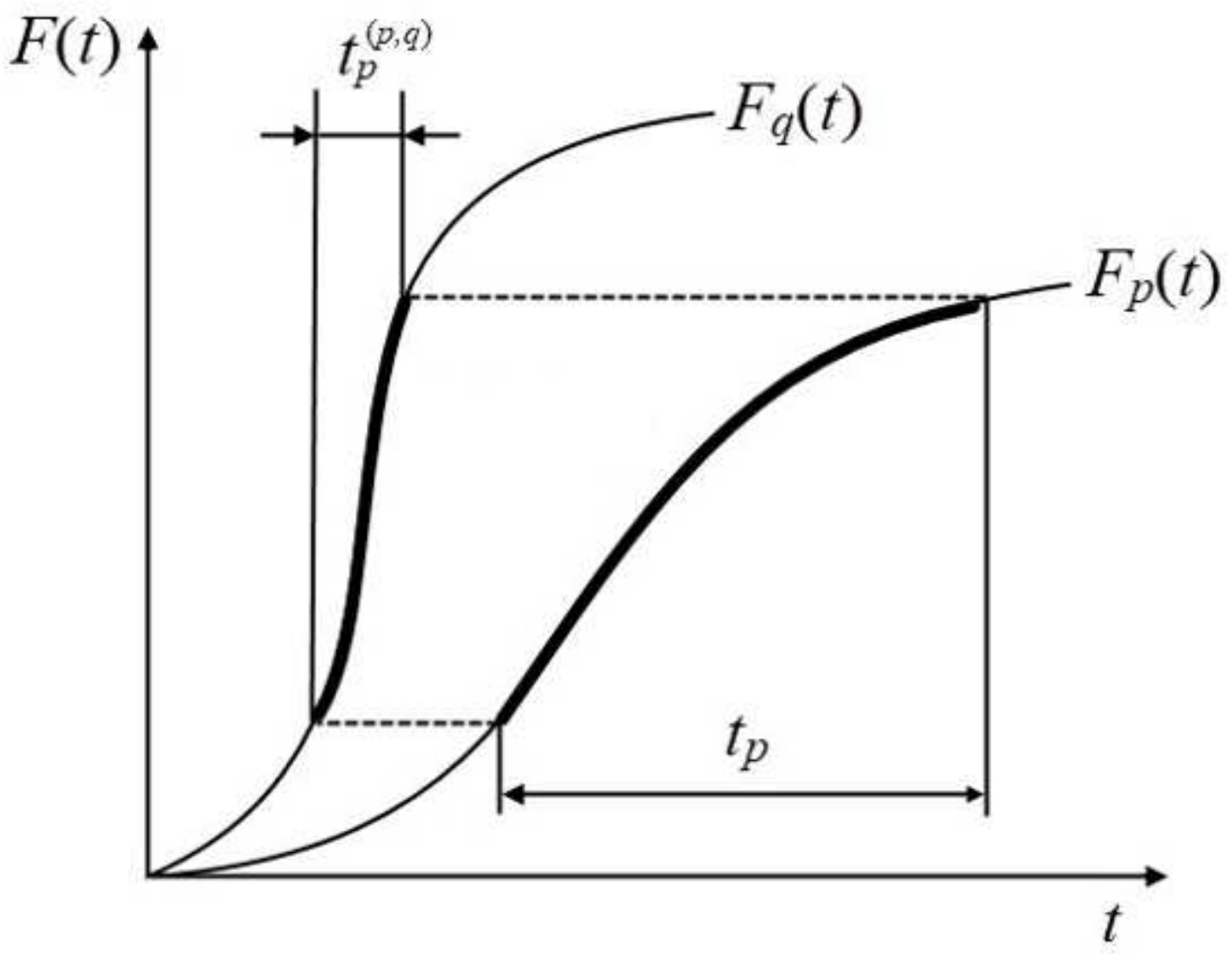

Figure 2 
Schematic diagram of equivalent conversion of Weibull distribution

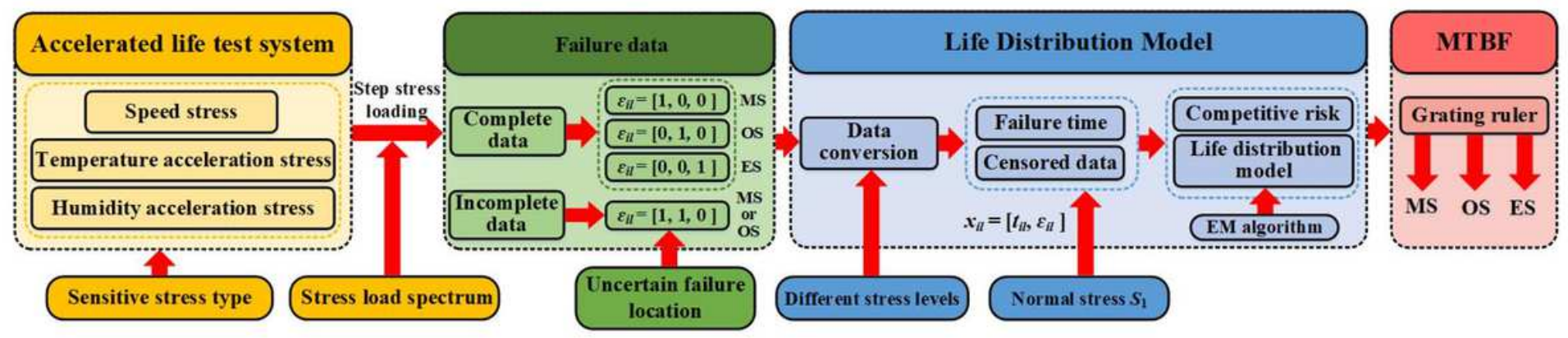

Figure 3

The process of the case analysis

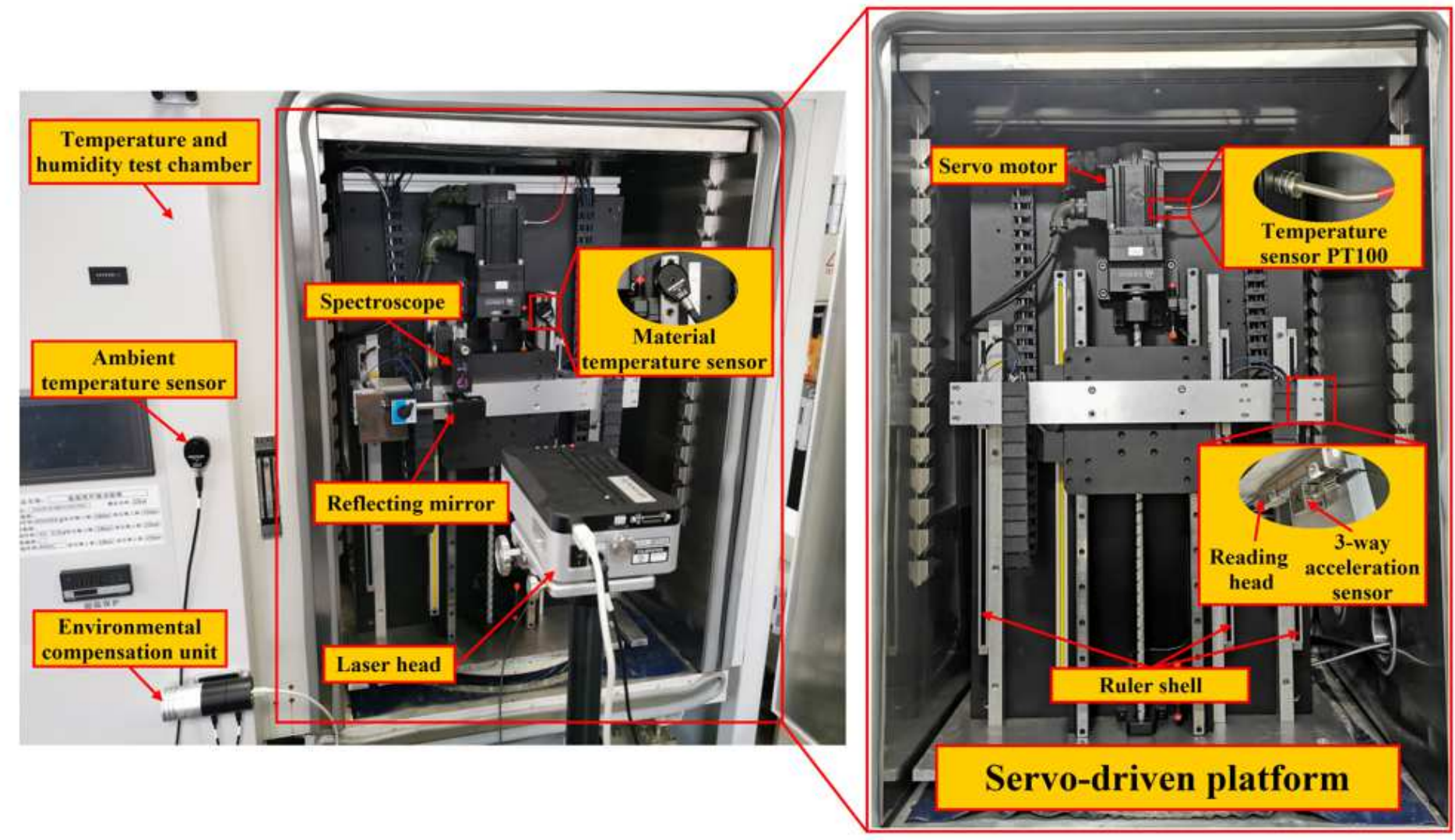

Figure 4

Accelerated life test device for grating ruler 


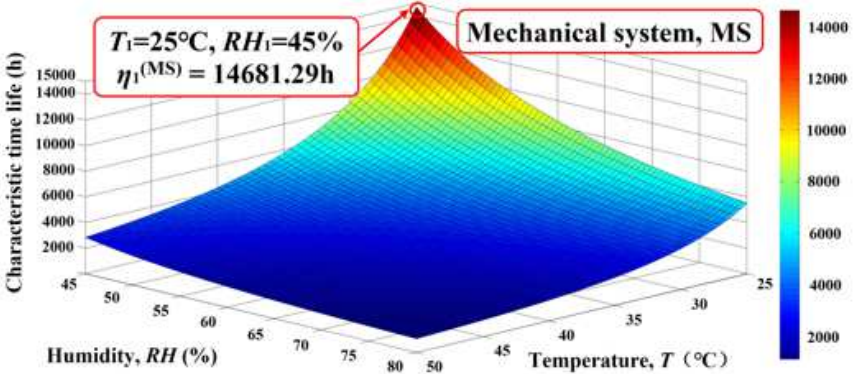

(a) Mechanical system

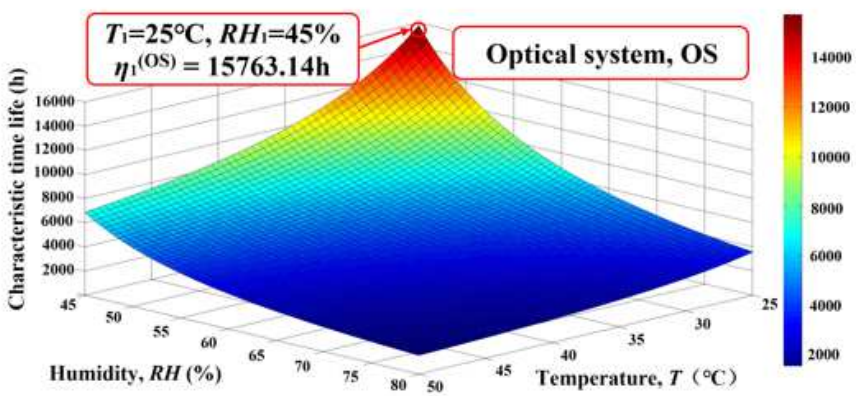

(b) Optical system

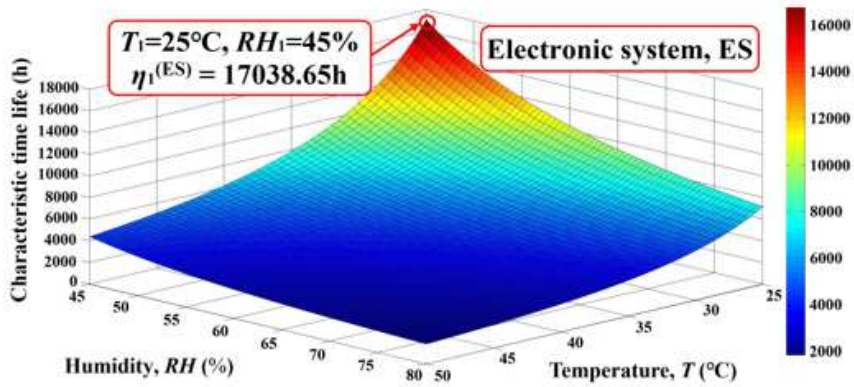

(c) Electronic system

\section{Figure 5}

Relationship between characteristic life and temperature and humidity stress

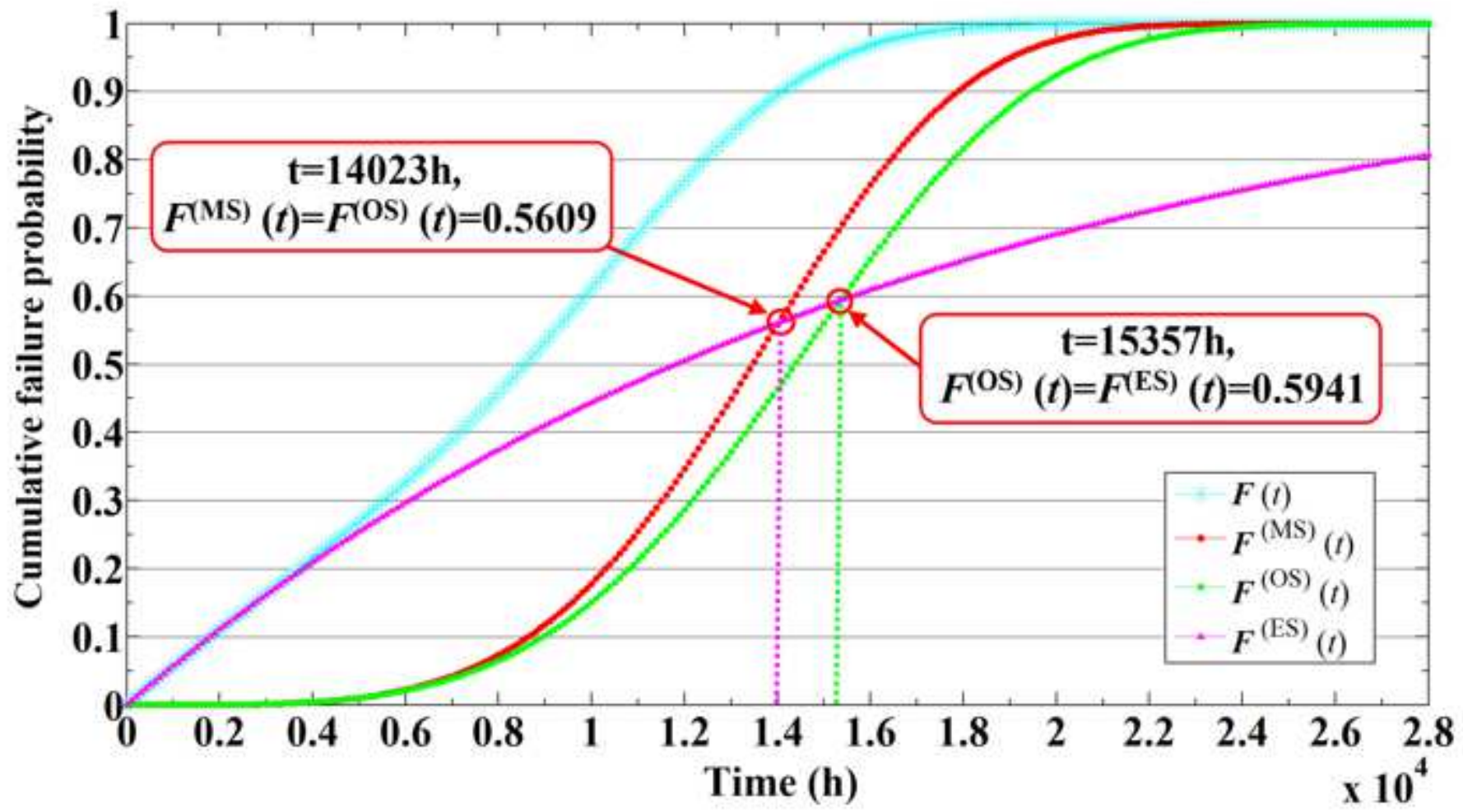

Figure 6 


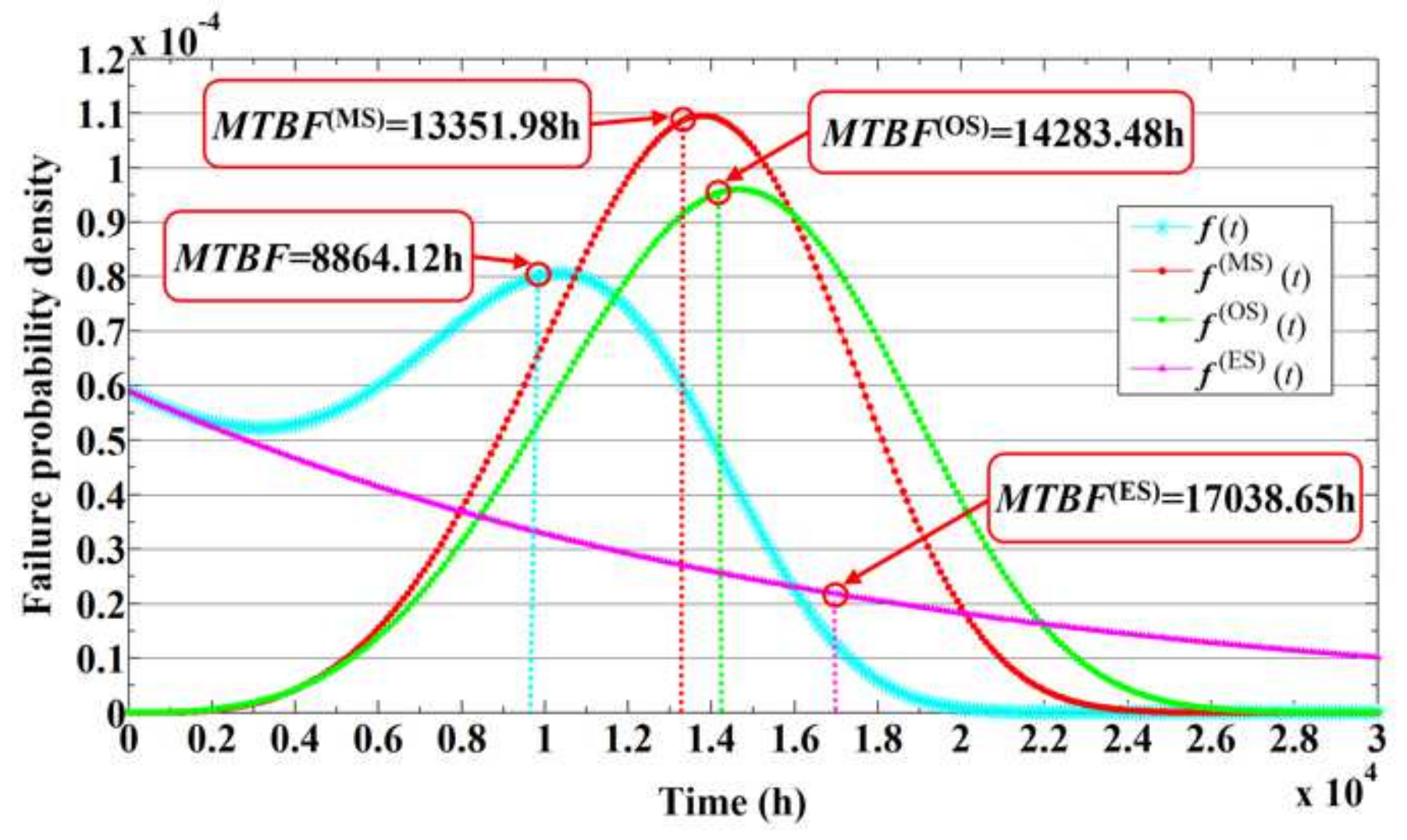

Figure 7

Probability density function based on competing risk model 